\title{
Una explicación desde el contexto y contenido de las políticas de salud al modelo híbrido y segmentado de atención primaria en salud en Bogotá
}

\author{
An explanation from context and content of health policies to a hybrid and segmented \\ primary health care model in Bogotá
}

Román Vega Romero', Jinneth Hernández Torres², Paola Andrea Mosquera Méndez³

' Doctor en Administración y Políticas de Salud. Profesor Asociado de la Pontificia Universidad Javeriana Bogotá, Colombia.

rrvega.romero@gmail.com

${ }^{2}$ Maestría en Sistemas de Salud; Investigadora en Evaluación de Sistemas y Programas de Salud y Atención Primaria en Salud por la Pontificia Universidad Javeriana Bogotá, Colombia.

jinnether@hotmail.com

${ }^{3}$ Maestría en Política Social; Candidata a Doctora en Salud Pública; Docente e Investigadora en sistemas de salud y atención primaria en salud, Pontificia Universidad Javeriana - Bogotá, Colombia.

paolamosquera@gmail.com
RESUMEN Este estudio respondió a la pregunta de cómo el contexto y el contenido de las políticas de salud de Colombia han influido en la implementación de un modelo híbrido y segmentado de atención primaria en salud en Bogotá en el período del 2004 al 2010, a pesar de haberse formulado otro de atención primaria en salud integral. Para ello se realizó un análisis de contenido de material documental, el cual evidenció que el modelo híbrido y segmentado resultante en la implementación está influido por la segmentación, fragmentación y orientación de mercado de aseguramiento y de prestación de servicios propia del sistema de salud colombiano y por la política de Gestión Social del Riesgo, los cuales, en conjunto, imponen limitaciones estructurales al diseño integral de atención primaria en salud formulado teóricamente por la autoridad sanitaria distrital.

PALAVRAS-CHAVE: Atención Primaria de Salud; Política de Salud; Determinantes Sociales; Implementación de Plan de Salud; Análisis Cualitativo.

\begin{abstract}
This study has answered the issue on how the context and content of Colombian health policies have influenced the primary health care model that was implemented in Bogotá, from 2004 to 2010, despite another primary care in integral health has already been created. Thus, a qualitative and documental analysis was carried out, which indicated that despite having formulated a CPHC conception, a hybrid and segmented approach emerged during the implementation. This approach is due to a segmented and fragmented health system driven by a market of insurance and health services and by a social policy based on social risk social management, which, together, impose structural limitations to the initial comprehensive formulation of the primary health care.
\end{abstract}

KEYWORDS: Primary Health Care; Health Policy; Social Determinants; Health Plan Implementation; Qualitative Analysis. 


\section{Introducción}

El objetivo de este estudio fue analizar la experiencia de atención primaria en salud (APS) en desarrollo en Bogotá desde el 2004 en términos de contrastar el enfoque de APS integral (APSI), formulado con el tipo de modelo implementado, y los factores contextuales y de contenido de las políticas que lo explican.

La APSI se fundamenta en la "Declaración de Alma Ata”, de 1978, y constituye una estrategia para organizar los sistemas de salud y la sociedad con el fin de recuperar, proteger y promover la salud para todos. Incluye la atención integral de la salud del individuo en el primer nivel, pero extiende sus acciones a la familia y a la comunidad y se articula con los niveles secundarios y terciarios del sistema de salud. No se reduce a la curación, rehabilitación, prevención y promoción dentro del sector salud, sino que compromete la acción de las comunidades y de otros sectores para afectar los determinantes sociales de la salud. La APSI incluye también los siguientes principios: respuesta a los más amplios determinantes de la salud; cobertura y accesibilidad universal según la necesidad; autocuidado y participación individual y comunitaria; acción intersectorial por la salud; y tecnología apropiada y uso costo-efectivo de los recursos disponibles.

De este enfoque se esperan los siguientes resultados: aumento del acceso a los servicios de salud y a otros recursos y servicios esenciales para la salud; reducción de la vulnerabilidad mediante el empoderamiento comunitario; reducción de la exposición de la población a riesgos, mediante cambios en los determinantes sociales y ambientales de la salud; mejoramiento de los mecanismos de participación comunitaria y de su capacidad de incidencia en las decisiones; aumento de las acciones de políticas con enfoque intersectorial; y mejora de la salud y de la equidad en salud de la población.

El Sistema General de Seguridad Social en Salud (SGSSS) colombiano se basa en un mercado de aseguramiento y prestación de servicios, con diferentes combinaciones público-privadas. En el SGSSS, las empresas aseguradoras administran los recursos procedentes de la afiliación individual, contratan la prestación de servicios y afilian los individuos en dos regímenes distintos: uno contributivo para quienes cotizan y otro subsidiado para quienes, según su condición de pobreza, reciben del Estado un subsidio total o parcial. Los dos regímenes tienen planes obligatorios de beneficios (POS) y unidades de pago por capitación, diferenciales para las acciones individuales. Por su parte, las acciones de salud pública contempladas en un plan de beneficios distinto y universal - Plan de Intervenciones Colectivas (PIC) - son dejadas bajo la responsabilidad de las autoridades públicas de salud, en el nivel local (HOMEDES; UGALDE, 2005, p. 210).

En el marco de este sistema de salud segmentado y fragmentado, el Gobierno Distrital de la ciudad de Bogotá decidió poner en práctica la estrategia de APSI. La propuesta de retomar eso surgió con la nueva política de salud iniciada en el 2004, la cual se orientó a garantizar el derecho a la salud con equidad, impactar los determinantes sociales y ambientales de la salud, mejorar el acceso a los servicios y reorientar el modelo de gestión y atención en salud. Se inició con el programa 'Salud a Su Hogar' (SASH) a finales del 2004 al que, con el cambio de gobierno a partir del 2008, se le denominó 'Salud a Su Casa' (SASC). Desde su diseño inicial y en el proceso posterior de implementación, el programa ha reivindicado un enfoque familiar y comunitario de acuerdo a lo formulado por la Organización Mundial de la Salud (OMS) (1978), a los atributos de la APS descritos por Starfield (2001) y a los valores, principios y elementos de la APS renovada (PAHO/WHO, 2007). La SASH o SASC ha tenido como propósito promover la atención integral en salud en el marco de la política social de Bogotá y mejorar la equidad en el acceso al SGSSS y a otros servicios y recursos relacionados con la salud.

La SAHS o SASC inicia su implementación en los territorios en desventaja social - estratos socioeconómicos uno y dos de la población ${ }^{1}$ - por medio de equipos básicos de salud (una enfermera, un médico, dos promotores y un técnico ambiental) que adscriben 1.200 familias. El equipo básico es apoyado por uno ampliado para cada localidad conformado por

' En Bogotá la población se clasifica en estratos socioeconómicos uno a seis, siendo el uno el más pobre y el seis el más rico. 
psicólogos, terapeutas físicas, nutricionista, odontólogo e higienistas orales y los técnicos y profesionales de entornos saludables. Ambos equipos son financiados por el PIC y operados por los hospitales de primer nivel de la red pública de servicios, las Empresas Sociales del Estado (ESE).

A pesar de que la orientación teórica y directrices generales de la SDS apuntaban a una APSI, el análisis del proceso de implementación realizado en algunas localidades permitió identificar que en la práctica se ha venido implementado un 'Modelo Segmentado e Híbrido' de APS, con predominio de un enfoque de gestión basado en las actividades de los gestores comunitarios que hacen actividades de prevención y promoción de la salud desde el PIC, que administra la autoridad sanitaria distrital, y de inducción de la demanda de actividades biomédicas de primer nivel incluidas en el plan obligatorio de salud administrado por las aseguradoras.

El modelo se ha denominado segmentado porque reproduce la segmentación del sistema de salud colombiano, e híbrido por entrecruzar elementos procedentes de tres tipos de APS: primero, algunos componentes de la APS de Alma Ata como la participación comunitaria y la acción intersectorial desarrolladas desde el PIC en los diferentes ámbito de vida cotidiana (escuela, trabajo, comunidad, familia e instituciones de protección) y el trabajo desarrollado por los gestores comunitarios desde el programa SASH/SASC; segundo, una especie de APS selectiva al operar, desde el primer nivel de atención, con intervenciones de los POS, que son costoefectivos, verticales y con un enfoque de atención administrada en el marco del contrato entre aseguradores y prestadores; y, tercero, el enfoque de atención médica básica tradicional desde los hospitales de primer nivel de atención.

Teniendo en cuenta que la reciente Ley $1.438 \mathrm{del}$ 2011 incluye la APS como estrategia central del sistema de salud de Colombia, se considera que el modelo implementado en Bogotá podría ser premonitorio de las dificultades para lograr su aplicación a nivel nacional. Bajo el supuesto de que el modelo 'híbrido y segmentado' es producto de las tensiones y contradicciones entre el contexto y el contenido de las políticas nacionales y distritales, el presente estudio pretendió identificar, desde la experiencia de implementación, los factores contextuales y del contenido de las políticas que explican como un diseño de APSI en lo teórico resulta en un modelo 'híbrido y segmentado’ en la práctica.

\section{Metodología}

Revisión histórica documental del contenido de las políticas sociales y de salud a los niveles nacional, distrital y local, orientada a explicar los factores que han influido en el modelo de APS híbrido resultante en Bogotá. Los documentos incluidos en la revisión (planes de desarrollo, normas legales, documentos de las Secretaría Distrital de Salud, entre otros) fueron valorados teniendo en cuenta que permitieron identificar los cambios históricos en la APS en Colombia, particularmente lo sucedido con la implementación de la Ley 100 de 1993 que reemplazó el Sistema Nacional de Salud creado en 1975, y la influencia de factores contextuales locales en el marco de la puesta en práctica de la APS en Bogotá con las políticas de los gobiernos de la ciudad desde el 2004, parcialmente alternativas al modelo neoliberal en desarrollo. A los documentos incluidos en la revisión, se les realizó un análisis de contenido extrayendo de ellos todo lo relacionado con los modelos de atención en salud, utilizando como categorías los atributos y los elementos de la APS (cuando ellos existieron), los modelos asistenciales en salud y otras categorías emergentes. Este estudio fue aprobado por el Comité de Ética de los programas de postgrado en Administración en Salud de la Facultad de Ciencias Económicas y Administrativas de la Pontificia Universidad Javeriana.

\section{Resultados y Discusión}

Como se describió, el modelo de APS diseñado buscaba reorientar la prestación de servicios de salud a nivel distrital a través de la conformación de equipos básicos de salud que desarrollaran acciones de promoción, prevención, curación y rehabilitación integradas e integrales a individuos, familias y comunidades. Este propósito se debilitó al reducirse SASH/SASC, principalmente a un programa de gestores comunitarios de salud cuyo papel principal ha sido la inducción de la demanda a 
los servicios de los centros tradicionales de atención médica, a los servicios de salud pública y a otros servicios sociales, y el desarrollo de actividades de información, educación y seguimiento para la promoción de la salud y prevención de la enfermedad, incluyendo algún énfasis en la promoción de la participación comunitaria y la acción intersectorial por la salud. El modelo resultante es, como se describe a continuación, el producto de las tensiones y las contradicciones entre el contexto y el contenido de las políticas nacionales y distritales, que se han dado a lo largo del tiempo.

En la historia de las políticas de salud de Colombia, dos reformas del sistema de salud han enmarcado la estrategia de APS luego de su promulgación en 'Alma Ata', en 1978. La primera reforma, el antiguo Sistema Nacional de Salud (SNS) constituido mediante el Decreto-Ley 056 de 1975, el cual integró funcionalmente la asistencia pública, el seguro social y la medicina privada y dio origen a un enfoque de APS segmentada de predominio 'primitivo', 'selectivo' y de atención de primer nivel y, segundo, el SGSSS con orientación de mercado constituido mediante la Ley 100 de 1993, actual plataforma de un enfoque de APS de predominio biomédico, selectivo y de atención administrada de primer nivel.

Cuatro concepciones de APS fueron desarrolladas por el gobierno de Colombia en el marco del antiguo SNS y hasta la formulación del modelo de aseguramiento con orientación de mercado en 1993. La primera fue la APS 'primitiva' de prestación de servicios mínimos de baja complejidad por parte de promotoras rurales y personal auxiliar de salud a partir de 1980, desarrollada desde el sector público, con el objetivo de ampliar la cobertura a grupos prioritarios de la población (MS, 1980). La segunda, la APS selectiva, promovida por el Banco Mundial y UNICEF a comienzos de los 80s (MS, 1983) y reforzada luego en el marco de las políticas económicas de ajuste estructural a través del Plan Nacional para la Supervivencia de la Infancia a mediados de los años 1980 (MS, 1985). La tercera fue el enfoque de
Sistemas Locales de Salud (SILOS) a partir del 1988, componente de la agenda de reforma del financiamiento y de la descentralización de los sistemas de salud en los países en desarrollo, promovida por el Banco Mundial (BM, 1987) y por la Organización Mundial de la Salud (WHO, 1987), y de las subsecuentes políticas de descentralización del Estado y de los servicios de salud a nivel local (DNP, 1987; MS/ACOMSAP, 1990), apoyadas con préstamos del Banco Mundial (MS, 1989, p. $58)^{2}$. La cuarta fue la vinculación de los SILOS con la promoción de la salud entre 1988 y comienzos de los años 1990, período en el cual la APS alcanza su máximo esplendor en Colombia acercándose más a los principios de la 'Declaración de Alma Ata', pero sin superar por completo la práctica médica convencional (MS, 1991; 1992a). Estos dos últimos desarrollos buscaban mejorar la articulación de la atención de las personas y del ambiente, la acción intersectorial por la salud, la participación social en las decisiones y la coordinación de los recursos del sector; organizar sobre una base geográfica y poblacional las intervenciones; estructurar la red local de servicios y ayudar a movilizar los recursos y la población hacia la salud; posibilitar la conexión entre los planes locales de salud y aquellos de desarrollos económico y social de los municipios; hacer eficaz la planificación estratégica y participativa, la gestión y el control en función de las necesidades locales de salud; y la formación del recurso humano desde las exigencias y las necesidades de la APS, la promoción de la salud y la medicina y salud familiar y comunitaria. Fue este el principal logro en materia de integralidad durante la experiencia de implementación y desarrollo de la APS en Colombia, antes de la formulación de la Ley 100 de 1993 (VEGA ROMERO et al., 2009).

El modelo de APS 'primitiva', 'selectiva' y de primer nivel de atención que se implementó con el SNS correspondió a uno nacional de desarrollo económico dependiente y basado en la sustitución de importaciones (MISAS ARANGO, 2002), que operó bajo la presión de un proceso de acumulación de capital que

2Según Giraldo (2007, p. 116): "El Banco Mundial ha jugado un papel central en las dos últimas décadas (el BID para el caso de América Latina en general se sujeta a los patrones del Banco Mundial), en razón de sus préstamos de ajuste estructural (structural adjustment lending), los cuales están atados a reformas institucionales y a la adopción de políticas públicas, entre las que se destaca la política social, en particular las reformas de los Sistemas de Protección Social". 
buscaba, desde los años 1970, reducir el gasto público y los costos de producción de una economía cada vez más oligopólica y globalizada en el marco de un proceso de intercambio desigual entre los países del Norte y del Sur (AMIN, 1978). Este enfoque de la política de acumulación de capital se desarrolló, sin embargo, en rivalidad con otro impulsado por el movimiento de los países no alineados y los sectores progresistas de las Naciones Unidas y del país (OPS, 2002, p. 221-257), en el marco de la lucha por un nuevo orden económico internacional, la autodeterminación de los pueblos, la paz con justicia social (COX, 1979) y un enfoque de desarrollo basado en la satisfacción de necesidades básicas (HOADLEY 1981; VEGA ROMERO; JARA NAVARRO, 2002, p. 81).

En el plano político, esto período de la historia colombiana se caracterizó por el inicio del proceso de desmantelamiento del Frente Nacional (FN), una coalición política bipartidista que rigió entre 1958 y 1974 para superar la violencia política de más de un siglo entre liberales y conservadores y garantizar el dominio hegemónico de los terratenientes y de las burguesías cafetera, industrial y comercial. El FN tuvo como expresión la alternancia en el gobierno cada cuatro años de los partidos liberal y conservador, el reparto burocrático paritario de los puestos del Estado entre los dos partidos hasta 1986, el enfoque presidencialista del ejercicio de gobierno y la exclusión de la participación ciudadana y de las fuerzas políticas y sociales alternativas del país. Este régimen político y económico constituye lo que algunos analistas han llamado del imperio de la democracia restringida en Colombia (OVIEDO HERNÁNDEZ, 1983).

Con la Ley 100 de 1993 (REPÚBLICA DE COLOMBIA, 1993), que reformó el anterior SNS y creó el actual SGSSS con economía de mercado, se terminó por completo con la experiencia de APS basada en la tradición de 'Alma Ata' (OPS, 2002) y con la APS selectiva de viejo tipo (CUETO, 2006, p. 27). El enfoque de APS asistencialista, neo-selectiva y de atención administrada de primer nivel que emergió bajo la Ley 100 se desarrolla en el contexto de un modelo de desarrollo económico aperturista y liberalizante (MISAS ARANGO, 2002), correspondiente al contenido de una política económica global controlada por oligopolios mundiales y trasladada desde el Norte hacia el Sur para generar excedentes económicos a través de la creación de un ejército global de mano de obra de reserva (BELLAMI FOSTER et al., 2011). Tal política económica ha tenido su contraparte en la iniciativa de varios países de la región, que han decidido poner en práctica reformas económicas, sociales y de políticas de salud progresistas (LAURELL, 2008, p. 83; ACOSTA et al., 2011, p. 1875), similares a las impulsadas por el 'Movimiento de los No Alineados' en los años 1970, aunque en otras condiciones históricas de procesos de integración y cooperación entre los países latinoamericanos, de crisis del modelo neoliberal y de deterioro de la hegemonía estadounidense (SECRETARÍA DE RELACIONES INTERNACIONALES DEL PARTIDO DE LOS TRABAJADORES DE BRASIL, 2011).

También en el plano político, a finales de los años 1980, termina el reparto burocrático paritario de los puestos del Estado entre los partidos liberal y conservador, se inicia la política de descentralización y reducción del tamaño del Estado, la elección popular de alcaldes y gobernadores y se producen los acuerdos de paz entre algunas fuerzas insurgentes y el gobierno liberal de Cesar Gaviria Trujillo, originando a la Constitución Política de 1991, que crea el llamado Estado Social de Derechos en un trasfondo de proyecto económico neoliberal (VEGA ROMERO; JARA NAVARRO, 2002, p. 81). Todo este proceso se dio en el marco de la continuidad del conflicto armado, social y político que tipifican los alzados en armas que no se acogen a los acuerdos de paz - lucha guerrillera que según sus actores (JIMÉNEZ, 2012, p. 18) tuvo su origen en el régimen de violencia, antidemocracia y exclusión política impuesto a partir del asesinado de Jorge Eliécer Gaitán, el 9 de abril de 1949, y con la puesta en práctica de la Doctrina de Seguridad Nacional por los gobiernos de Estados Unidos en el marco de la Guerra Fría luego de la Segunda Guerra Mundial.

El proceso de asimilación de la APS en el marco de la política neoliberal de Colombia no se ha dado sin contratiempos. Con el proceso de puesta en práctica de la Ley 100 de 1993, no sólo no se materializó el 'Plan Nacional de Atención Primaria' incluido en el PND del gobierno de Cesar Gaviria Trujillo, sino que se comenzó a desmantelar la experiencia simplificada 
y limitada de APS introducida desde lo comienzo de los años 1980 (JARAMILLO SALAZAR, 1980; MS, 1981) y los desarrollos posteriores alcanzados mediante la descentralización de la atención básica, la constitución de los SILOS y la introducción de la Promoción de la Salud con la Ley 10 de 1990 (OPS, 1988; OPS/ OMS/SSPMC, 1990; MS, 1992b).

La APS comenzó a existir como estrategia normativa vinculante del conjunto del actual SGSSS sólo a partir de la aprobación de la Ley 1.438, de 2011 (REPÚBLICA DE COLOMBIA, 2011). Previo a esta Ley, la APS había sido una iniciativa marginal y discrecional de la política de prestación de servicios y salud pública (MPS, 2005; 2007) y de la actividad de algunas autoridades territoriales de salud y EPS 3 .

La mayor parte de las pocas experiencias de atención primaria desarrolladas por las EPS han estado basadas en modelos biomédicos de manejo de riesgos individuales y de asistencia médica, con enfoque de atención administrada. Estos modelos han usado más la atención médica de primer nivel y la domiciliaria y la medicina familiar para controlar los costos de la atención de las enfermedades, especialmente de las crónicas y de alto costo para facilitar el acceso al sistema de salud, garantizar la atención integral de la población según necesidades y propiciar el control de los riesgos de salud y sus causas, los determinantes sociales de la salud.

La reducción de la salud pública convencional a sólo aquellos bienes públicos con altas externalidades positivas introducidos en el Plan de Atención Básica (PAB) a cargo del Estado - luego denominado PIC -, y el empaquetamiento de todas las demás actividades de atención médica individual consideradas bienes privados o mixtos en el Plan Obligatorio de Salud (POS) a cargo de las EPS y por los cuales se debería pagar individualmente una cotización, fue parte de la política que llevó a la implantación de un mercado de aseguramiento y de prestación de servicios que, aunque 'regulado', a la postre produjo la negación del territorio (en su doble dimensión poblacional y de lugar) como referente para la formulación de políticas públicas y el diseño de intervenciones en salud, la profundización de la segmentación y fragmentación del sistema de salud, la liquidación del enfoque de APS que se venía desarrollando hasta principios de los años 1990, y centró los esfuerzos del gobierno en los aspectos organizacionales, financieros y gerenciales de la implementación de la reforma de 1993 (OPS, 2002).

Como se sabe, el tipo actual de enfoque de la APS depende en gran parte del contenido de las políticas sanitarias de las agencias de las Naciones Unidas y de las decisiones de las élites de Colombia. Aunque algunas de esas políticas han tenido como propósito mejorar la cobertura de los servicios de salud y la salud de la población, han sido fundamentalmente funcionales a políticas macroeconómicas orientadas tanto a controlar el gasto público en salud (MAHLER, 1975; FONDO DE NACIONES UNIDAS PARA LA INFANCIA, ORGANIZACIÓN MUNDIAL DE LA SALUD, 1975; BM, 1975), mediante el uso de tecnologías de atención en salud de bajo costo, como a promover el lucro y la acumulación de capital, mediante el aprovechamiento de las ventajas comparativas de los bajos salarios y la formación de capital humano de bajo costo en los países en desarrollo, enfrentar las crisis económicas de acumulación mediante políticas de ajuste, e implementar estrategias de globalización neoliberal de la producción del capital multinacional a partir de los años 1980 (BELLAMI FOSTER et al., 2011; HYMER, 1979, p. 41).

En el caso de Colombia, la política que dio origen al SGSSS se ha llevado a cabo en el contexto del proceso de implementación de las reformas económicas y sociales neoliberales, las cuales dieron origen a la simbiosis entre apertura y liberalización económica y lo que hoy se llama el 'Estado Social de Derecho'. El contenido del modelo de crecimiento económico de los Planes Nacionales de Desarrollo desde el Gobierno de Cesar Gaviria Trujillo hasta el actual de Juan Manuel Santos, y aquello de las sucesivas reformas institucionales en seguridad social y salud que de ellos se derivan,

2 En el Encuentro Nacional de APS realizado entre el 25 al 28 de mayo de 2005 para discutir la propuesta de APS renovada promovida por la OPS se descubrió que la APS se había mantenido viva en la memoria de los funcionarios de salud, de las universidades y de las comunidades, y que se venía desarrollando más allá de lo previsto. Ejemplos de ello eran Bogotá, y algunos casos en Santander, Valle, Antioquia Magdalena Cartagena, Barraquilla, Nariño y Cauca, y en EPS como Servicio Occidental de Salud y Mallamás EPS-Indígena. 
reflejan los fundamentos del Consenso de Washington I y II (GIRALDO, 2007) ${ }^{4}$, las políticas impulsadas por el Banco Mundial (BM, 1993) y, en lo nacional, los acuerdos sobre derechos sociales, económicos y culturales que dieron salida parcial al conflicto armado interno del país y que fueron materializados en la Constitución Política de 1991 (MISAS ARANGO, 2002; VEGA ROMERO; JARA NAVARRO, 2002; GIRALDO, 2007).

La contradicción en el distinto contenido de ambas perspectivas al expresarse en el discurso y en la práctica de los gobiernos sobre las políticas sociales y de salud es una de las mayores fuentes de tensión y conflicto que ha gravitado entre, de una parte, la búsqueda de universalidad, integralidad y equidad de las políticas sociales y de salud que han defendido las fuerzas progresistas y democráticas y, de otra, la preocupación de los neoliberales por los problemas inflacionarios y fiscales, el control de los costos laborales y de la atención de la enfermedad, la mercantilización y focalización del manejo de los riesgos sociales, y la privatización y empresarialización creciente de las vidas social e individual en aras de la rentabilidad económica.

Según la perspectiva reformista impuesta por el nuevo bloque hegemónico de poder constituido por conglomerados económicos productores de bienes no transables, empresas transnacionales y del capital financiero que reemplazó a la vieja hegemonía de cafeteros, industriales y terratenientes coaligada alrededor del Modelo de Sustitución de Importaciones (MISAS ARANGO, 2002), el nuevo modo de regulación adoptado a partir de los principios del 'Consenso de Washington I' impulsó dos 'nuevas' formas de intervención económica del Estado para suscitar el crecimiento y el desarrollo, que les beneficiaría con la privatización de empresas y servicios estatales, la apertura y la financiarización: promover los mercados y las competencias interna y externa en un marco de apertura económica y de liberalización; alcanzar una forma óptima de intervención en la economía a través de una acción transitoria, selectiva y complementaria orientada a corregir las distorsiones del mercado ${ }^{5}$, concentrando la inversión pública o la combinación de inversión público-privada en actividades que generaran externalidades positivas, que evitaran las negativas o que indujeran rentabilidad económica creciente de la inversión privada, por ejemplo, en capital humano, seguridad, infraestructura física y de transporte, adecuación de tierras, tecnología y descontaminación de recursos ambientales. Complementariamente, la acción social del Estado debería guiarse por los siguientes criterios: crear las condiciones para aumentar las capacidades de generación de ingresos y de calidad del capital humano de los grupos más desfavorecidos de la población, mejorando simultáneamente las oportunidades para su inserción en el mercado; mejorar la equidad en la distribución del capital humano ${ }^{6}$ como estrategia para perfeccionar la equidad en la distribución del producto y de los ingresos $^{7}$ y favorecer el crecimiento y el desarrollo, lo que facilitaría una intervención estatal limitada a las externalidades del capital humano que garantizara la libertad individual, la eficiencia y la equidad.

Para alcanzar este último objetivo, bastaría aumentar y redistribuir la educación, la salud y la nutrición - una cesta limitada de derechos -, mediante acciones focalizadas (no universalistas) en los grupos que requirieran mayor consideración ${ }^{8}$. Lo anterior significaría que el resto de la población debería garantizarse

\footnotetext{
${ }^{4}$ De esos acuerdos hicieron parte agencias multilaterales como el Banco Mundial, el Fondo Monetario Internacional, el Banco Interamericano de Desarrollo y la Reserva Federal.

${ }^{5}$ Fallos del mercado explicables por bienes públicos o con externalidades que no permiten un adecuado cálculo costo-beneficio en las decisiones de los agentes.

${ }^{6}$ El capital humano emerge como un "concepto clave en la política social porque define las capacidades productivas de la persona en función de su dotación en educación y salud, principalmente. Desde este punto de vista los pobres son poco productivos porque tienen una baja dotación en capital humano, y el gasto social debe dirigirse a proveerles una dotación mínima". (GIRALDO, 2007, p. 21)

${ }^{7}$ El Estado de Bienestar Europeo supuso un pacto entre capital (liberalismo) y trabajo (socialismo) para la repartición de los incrementos de productividad (redistribución) "que permitía la ampliación del mercado interno, favorecido por políticas keynesianas" (GIRALDO, 2007, p. 21). En la concepción del PND, no hay una redistribución de los productos a partir de una estrategia de aumento de la productividad del trabajo como en el Estado de Bienestar, sino otra de aumento de la productividad del trabajo de los pobres, de lo cual derivaría las mejoras sociales que éstos obtengan.

${ }^{8}$ Según Giraldo (2007, p. 23): "Los pobres y los no pobres están sujetos al riesgo, sólo que en el caso de los primeros ello se traduce en ‘vulnerabilidad'y por tanto deben ser objeto de políticas asistencialistas (focalizadas: esto es mío), mientras que los segundos tienen la opción de de comprar un seguro para mitigar dicho riesgo. De manera que aquí claramente la política hacia los pobres se diferencia de los 'no pobres'. Para los últimos la propuesta se basa en el aseguramiento". Es de anotar que en el caso colombiano, en sentido estricto, el aseguramiento sólo opera para el régimen contributivo porque lo subsidiado denota una política de asistencia pública.
} 
por sí misma su bienestar, deseos y aspiraciones a través de los mecanismos del mercado. No sería en función de la solidaridad o de derecho social universal alguno que el Estado debería intervenir para bien de los excluidos y desprotegidos, sino en función de mejorar las capacidades y las oportunidades de éstos para insertarse en el mercado, que no arriesgaría la modificación de la estructura social y de poder económico/político establecido, ni pondría en peligro las libertades individuales.

Énfasis especial de las políticas neoliberales ha sido el control del gasto público. En el caso de salud y educación, el gasto del Gobierno es asumido como una inversión en capital humano y en consumo, pero éste tiene que ser focalizado en los más pobres para satisfacer sus necesidades básicas y ayudarles a aprovechar las oportunidades del mercado. En este sentido, el gasto debe ser hecho especialmente en programas de educación primaria y de atención primaria, los cuales generan capacidades y vigor para la inserción en el mercado laboral y para garantizar cierto nivel de productividad del trabajo. También es creencia generalizada que las empresas estatales, y aún los servicios públicos, deben ser privatizados para que sus manejos y las competencias los hagan más eficientes, sobre todo porque se considera que al tener el sector privado el lucro como fin, éste se constituye en un incentivo para lograr la eficiencia. Estrechamente relacionada con esta medida, estaría la desregulación de la inversión foránea de capitales, del establecimiento de empresas, el retorno de las ganancias al sitio de origen del capital invertido y la flexibilización y desregulación del trabajo.

En contraposición al enfoque de APS funcional del negocio de la salud y de la acumulación de capital, que busca servir de instrumento para controlar el costo de la atención de la enfermedad y para la formación de capital humano a bajos costos crecidos discrecionalmente en el marco del SGSSS hasta el 2010, en el caso de Bogotá, y como parte de la política orientada a promover y garantizar el derecho a la salud del bloque político de centroizquierda que llegó al Gobierno de la ciudad por primera vez en el año 2004, el gobierno distrital formuló una propuesta de APS integral, con enfoque familiar y comunitario (AMB/SDS, 2004), que en la práctica, dado el peso de la política nacional de salud con orientación de mercado, el modelo neoliberal de desarrollo económico y la ausencia de un Estado social y democrático verdadero, resultó en un 'modelo híbrido y segmentado de APS' que conjuga elementos del enfoque integral con el de atención administrada de primer nivel y selectivo (biomédico).

\section{Conclusiones}

El 'modelo híbrido y segmentado' de APS desarrollado en Bogotá parece ser el resultado de la tensión permanente entre los factores del contexto, contenido de la política económica, social y de salud, y del proceso de implementación, que reflejan intereses contrapuestos de los actores del sistema de salud a diferentes niveles. El enfoque neoliberal de las políticas social y de salud a nivel nacional, basadas en la gestión social del riesgo, la atención administrada en salud, la desterritorialización de las acciones, la lógica de rentabilidad financiera del SGSSS y la discrecionalidad y la falta de relevancia dada a la APS en el mismo han impuesto limitaciones estructurales para que se pueda desarrollar un enfoque de APSI.

Reconociendo que la APS ha sido una prioridad para los dos últimos gobiernos de la ciudad de Bogotá y que se ha tenido que desarrollar en este contexto adverso, también se perciben algunas debilidades desde la SDS para su implementación: la complejidad y la confusión conceptual en su relación con la Estrategia Promocional de Calidad de Vida y Salud (SDS, 2006); la rigidez de los lineamientos de administración del PIC desde la SDS; los vacíos en la generación de mecanismos y políticas laborales que disminuyan la inestabilidad y rotación del personal; la discontinuidad en el proceso de formación del recurso humano; y la debilidad institucional en la construcción de intersectorialidad.

En últimas, tal modelo de APS al estar estructuralmente determinado por el contenido de mercado de la reforma de 1993 del sistema de salud y de otras políticas económicas y sociales parece indicar las dificultades que la nueva Ley 1.438 encontrará en la aplicación de la APS en Colombia. Esta experiencia es también una enseńanza para otros países sobre la aparente incompatibilidad entre reformas de de mercado de los sistemas de salud y la APSI, actualmente recomendada como estrategia clave para fortalecer sistemas de salud que busquen contribuir a la equidad social en salud. 


\section{Referências}

ACOSTA RAMÍREZ, N. et al. Comprehensive Primary Health Care in South America: contexts, achievements and policy implications. Cadernos de Saúde Pública, Rio de Janeiro, v. 27, n. 10, p. 1875-1890, 2011.

ALCALDÍA MAYOR DE BOGOTÁ. SECRETARÍA DISTRITAL DE SALUD (AMB/SDS). Salud a Su Hogar. Un modelo de Atención Primaria en Salud para garantizar el derecho a la salud en Bogotá. Bogotá: Secretaria Salud, 2004.

AMIN, S. The Law of Value and Historical Materialism. New York: Monthly Review Press, 1978, p. 1-2.

BANCO MUNDIAL (BM). Informe sobre el desarrollo mundial 1993: invertir en salud. Washington, D.C.: Banco Mundial, 1993.

El financiamiento de los servicios de salud en los países en desarrollo. Una agenda para la reforma. Washington, D.C.: Banco Mundial, 1987.

Salud: documento de política sectorial. Washington, D.C. Banco Mundial, 1975.

BELLAMY FOSTER, J.; MCCHESNEY, R.W.; JAMIL JONNA, R. The Global Reserve Army of Labor and the New Imperialism. Monthly Review, v. 63, n. 6, 2011. Disponível em: <http://monthlyreview. org/2011/11/01/the-global-reserve-army-of-labor-and-the-new imperialism>. Acesso em 01 mar. 2012.

COX, R.W. Ideologies and the new international economic order: reflections on some recent literature. International Organization, Madison, v. 33, p. 257-302, 1979.

CUETO, M. Los orígenes de la Atención Primaria de Salud y la Atención Primaria Selectiva de Salud. In: CUETO, M.; MESÍA, V.Z. (Ed). Historia, salud y globalización. Lima: IEP, UPCH, 2006, p. 27-58.

DEPARTAMENTO NACIONAL DE PLANEACIÓN (DNP). Plan de economía Social. Planes y programas de desarrollo económico y social, 1987-1990. Bogotá: DNP, 1987.

FONDO DE NACIONES UNIDAS PARA LA INFANCIA; ORGANIZACIÓN MUNDIAL DE LA SALUD. Necesidades sanitarias básicas de los países en desarrollo: posibles soluciones. Crónica de la OMS, v. 29, 1975, p. 179-200.

GIRALDO, C. ¿Protección o desprotección social? Bogotá, D.C.: Fundación CESDE/ Universidad Nacional de Colombia,2007.

HOADLEY, J.S. The rise and fall of the basic needs approach. Cooperation and Conflict, v. 16, 1981.

HOMEDES, N.; UGALDE, A. Neoliberal reforms in health services in Latin America: a critical view from two case studies. Revista Panamericana de Salud Pública, Washington, v. 17, n. 3, p. 210-220, 2005.
HYMER, S. H. The Multinational Corporation. Cambridge: Cambridge University Press, 1979.

JARAMILLO SALAZAR, A. Informe del Ministerio de Salud al Congreso de la República. Bogotá: MS, 1980.

JIMÉNEZ, T. Carta a Medófilo Medina. In: UN DEBATE NECESARIO: Diálogo epistolar - Colección Batalla de Ideas. Bogotá, D.C: Fundación Semanario Voz, 2012, p. 18-39.

LAURELL, A.C. Sacando a las reformas progresistas de la sombra. [Editorial]. Medicina Social, v. 3, n. 2, 2008, p. 83-86. Disponível em: <www.medicinasocial.info>. Acesso em: 01 mar. 2012.

MAHLER, H. Estrategias sanitarias en un mundo en transformación. Crónica de la OMS, v. 29, 1975.

MINISTERIO DE LA PROTECCIÓN SOCIAL (MPS). Decreto 3039. Por el cual se adopta el Plan Nacional de Salud Pública 2007-2010. Bogotá, D.C.: MPS, 2007.

Política Nacional de Prestación de Servicios de Salud. Bogotá, D.C.: MPS, 2005.

MINISTERIO DE SALUD (MS). Reorganización del Sistema Nacional de Salud. Una respuesta al cambio social. Memoria analítica de la Ley 10 de 1990. 2 Vols. Bogotá: MS/ACOMSAP, 1990.

Salud para Todos. Memorias 1991-1992. Santa Fe de Bogotá: MS, 1992a.

Descentralización y Municipios Saludables para un nuevo departamento. Bogotá: Gráficas Macondo, 1992b.

La revolución de la salud. Santa Fe de Bogotá: MS, 1991.

Memoria al congreso 1988-1989. Bogotá: MS, 1989.

Plan Nacional para la Supervivencia de la Infancia y el Desarrollo Infantil. Bogotá: Ministerio de Salud, 1985 (Documento no publicado).

Memoria al Congreso 1982-1983. Bogotá: MS, 1983.

Informe al Honorable Congreso de la República. 2 Tomos. Bogotá: MS, Instituto Nacional de Salud, 1981.

Informe del Ministro de Salud, Alfonso Jaramillo Salazar, al Congreso de la República. Bogotá: MS, 1980.

MISAS ARANGO, G. La Ruptura de los 90. Del gradualismo al colapso. Bogotá: Universidad Nacional de Colombia, 2002.

ORGANIZACIÓN MUNDIAL DE LA SALUD (OMS). Atención Primaria de Salud. Informe de la Conferencia Internacional sobre Atención Primaria de Salud. Alma-Ata, URSS. Ginebra: Serie Salud para Todos 1, 1978. 
ORGANIZACIÓN PANAMERICANA DE LA SALUD (OPS). La Organización Panamericana de la Salud y el Estado colombiano: cien años de historia 1902-2002. Bogotá, D.C.: Carrera 7, 2002.

Desarrollo y Fortalecimiento de los Sistemas Locales de Salud en la Transformación de los Sistemas Nacionales de Salud. Documento CD 33/14, Res. XV - XXXIII del Comité Directivo, Washington, D.C.: OPS, 1988.

ORGANIZACIÓN PANAMERICANA DE LA SALUD; ORGANIZACIÓN MUNDIAL DE LA SALUD; SECRETARÍA DE SALUD PÚBLICA MUNICIPAL DE CALI (OPS/OMS/SSPMC). Desarrollo de los Sistemas Locales de Salud en los Países de la Sub-región Andina. Cali: OPS/ OMS, 1990.

OVIEDO HERNÁNDEZ, A.A. Colombia, democracia restringida o apertura democrática. Bogotá: Colecciones CEIS, 1983.

PAN AMERICAN HEALTH ORGANIZATION; WORLD HEALTH ORGANIZATION (PAHO/WHO). Renewing Primary Health Care in the Americas. A Position Paper of the Pan American Health Organization /World Health Organization. Washington, D.C.: PAHO, 2007.

REPÚBLICA DE COLOMBIA. Congreso de la República. Ley 100 de 1993. Por la cual se crea el Sistema de Seguridad Social Integral y se dictan otras disposiciones. Diario Oficial, jueves 23 de diciembre, Año 129, n. 41.148, p. 1-37.

Ley 1438 de 2011. Por medio de la cual se reforma el Sistema General de Seguridad Social en Salud y se dictan otras disposiciones. Bogotá, D.C.: 19 de enero de 2011.
SECRETARÍA DISTRITAL DE SALUD (SDS). Lineamientos de salud pública 2006. Bogotá: Dirección de Salud Pública, 2006. No publicado.

SECRETARÍA DE RELACIONES INTERNACIONALES DEL PARTIDO DE LOS TRABAJADORES DE BRASIL. Foro de São Paulo. XVII Encontro do Foro de São Paulo (Resoluciones), Manágua, maio de 2011. Disponible en: <www.pt.org.br>. Acesso em: 15 mar. 2012.

STARFIELD, B. Atención Primaria. Equilibrio entre necesidades de salud, servicios y tecnología. Barcelona: MASSON, 2001.

VEGA ROMERO, R.R. Los orígenes y desarrollos de la APS en Colombia. In: VEGA ROMERO, R.R. et al. Atención Primaria de Salud. Estrategia para la transformación del Sistema de Salud y el logro de la equidad en salud. Bogotá, D.C.: Unión Gráfica, 2009.

VEGA ROMERO, R.; JARA NAVARRO, M.I. Década de los noventa: discurso de la focalización de subsidios en salud. Revista Gerencia y Políticas de Salud, v. 2, p. 81-112, 2002.

WORLD HEALTH ORGANIZATION (WHO). Decentralization and health for all strategy. Geneva: WHO, 1987.

Recibido para publicação em Abril/2012

Versão definitiva em Agosto/2012

Suporte financiero: Programa de investigación en Salud Global Teasdale-Corti - un programa de investigación

Conflito de interesse: inexistente 